\title{
Short-wave photodetectors based on fine grain-sized poly-Si films
}

\author{
F.G. Agaev \\ Azerbaijan National Aerospace Agency, Baku, 370159, Azerbaijan
}

\begin{abstract}
Photoelectric properties of polycrystalline silicon films under illumination were investigated. It was shown that polysilicon films of fine grain sizes may be used as short-wave photodetectors, due to presence of shallow $p$ - $n$ junctions at their grain boundaries.
\end{abstract}

Keywords: polysilicon films, photodetector, spectral sensitivity.

Paper received 28.12.00; revised manuscript received 21.01.01; accepted for publication 16.02.01.

In recent years an increasing interest to high-speed shortwave photodetectors is observed. It is known that sensitivity in this spectral range is reached by using extremely shallow $p-n$ junctions, the formation of which makes some difficulties from the technological viewpoint. For this reason the lightly doped poly-Si films are of interest, due to presence of deep traps at their grain boundaries. These conditions provide formation of space charge regions the parameters of which could be controlled at a given concentration by changing grain sizes [1]. Taking into account the grain sizes and doping concentration level ratio in polycrystalline semiconductors, the following cases can be realized: i) the grain conductivity type corresponds to that of impurity (high concentration, large grain size), either ii) the depleted Schottky layers or the regions with opposite conductivity types are formed at the grain boundaries (average concentration and grain size) and, at last, iii) at low impurity content and small grain sizes, the space charge region can spread over the whole grain, and the conduction is realized through inter-grain spaces. The lightly doped space charge regions with small grain sizes can act as shallow $p-n$ junctions at the grain boundaries of poly-Si films enabling their use as photodetectors. Their technology has advantages of excluding the diffusion and ion doping steps.

The photon - induced current properties of poly-Si films, formed in the process of epitaxial growth of singlecrystalline silicon films of $n$-type conductivity on locally masked silicon substrates were investigated in this paper. The film thickness and resistivity $(\rho)$ were $6 \mu \mathrm{m}$ and $0.2 \Omega \cdot \mathrm{cm}$, respectively. The ohmic contacts to poly-Si were formed by vacuum deposition of $\mathrm{Al}$ on the previously formed $n^{+}-$regions.
The films with average grain sizes of 0.5 and $5 \mu \mathrm{m}$, as well as linear sizes $200 \times 20,400 \times 40$ and $600 \times 60 \mu \mathrm{m}^{2}$, were investigated. The measurements of poly-Si films changes under the influence of illumination showed that for both fine and large grain sized films the open circuit voltage $U_{i}$ was in proportion to the film length, and the short circuit current $I_{o S}$ did not depend on the film length and was determined by its width and grain sizes within the film.

From the current-voltage characteristics of the element with fine grain sized structure and area of $3.6 \times$ $\times 10^{-2} \mathrm{~mm}^{2}$, it was seen that the ratings of $U_{i}$ and $I_{o s}$ were $140 \mathrm{mV}$ and $10 \mu \mathrm{A}$, respectively, and the efficiency was of the order of $5 \%$, while for the large grain sized films the corresponding values were $170 \mathrm{mV}, 15 \mu \mathrm{A}$ and $7 \%$.

It is known that after the electrical forming consisting in forcing current pulses (300 $\mathrm{mA})$ through the film, an effect of bistable switching could be observed in poly-Si films [2].

In electrically formed films being in a high-resistance state, $U_{i}$ and $I_{o s}$ were 3 to 4 times higher. The assumption was made that the abrupt increase of poly-Si photocells efficiency is connected with the anomalous photoelectric voltage effect conditioned by the photo-EMF summing that arises in space charge regions of grain boundaries after the electrical forming. According to the physical mechanism of switching suggested in [3], the role of electrical forming was in thermal breakdown of one of the space charge regions near grain boundaries which at a given polarity of bias applied was reverse biased. This summing of photo-EMF arising at the opposite grain boundaries takes place in a way described in [4] for the periodic structures of $p-n$ junctions connected to each 


\section{F.G. Agaev: Short-wave photodetectors based on fine grain size poly-Si films}

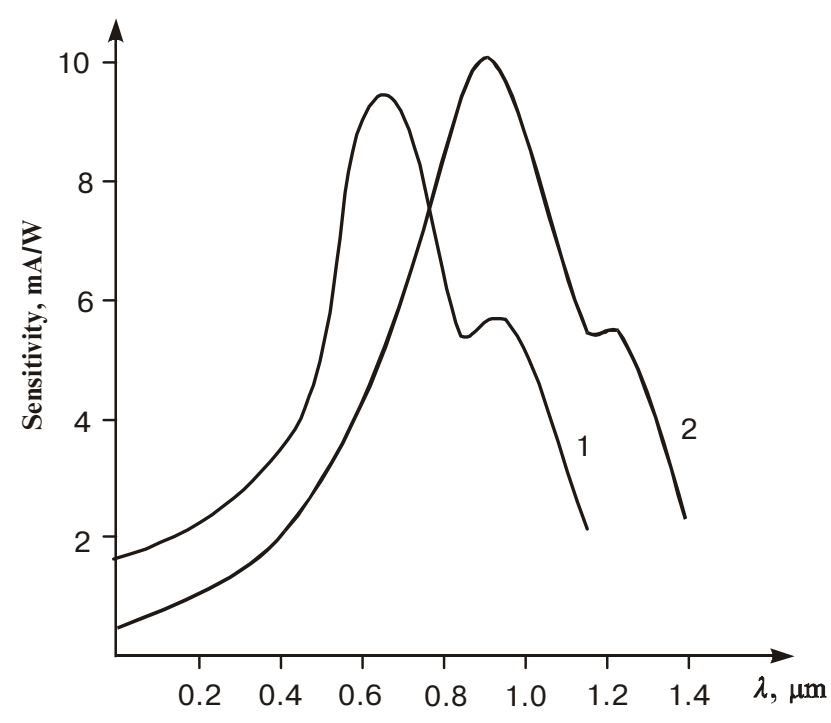

Fig. 1. Spectral dependencies of an absolute sensitivity of electricaly formed poly-Si films. The curve 1 corresponds to fine grain sized films, and curve 2 to large grain sized films.

other by metal jumper. As a result of the electrical forming, the efficiency of the fine grain sized poly-Si films with the incident light power being $93 \mathrm{~mW} / \mathrm{cm}$ was increased from 5 to $15 \%$.

The electrically formed poly-Si films were also measured in a light irradiation pulse mode. The light pulses were supplied by the light- emitting diode (LED) AL106 with the radiation wavelength and power being $0.9 \mu \mathrm{m}$ and $2 \mathrm{~mW}$, respectively. The LED was fed by the generator G5-54.The readings from the oscilloscope C1-65 showed the value of the output pulse height from the polySi film being $150 \mathrm{mV}$. The form of the output pulses from the poly-Si film was similar to that of the rectangular pulses, supplied by the generator to LED up to a maximum frequency being of the order of $90 \mathrm{kHz}$.
The absolute sensitivity spectral dependencies for the fine (curve 1) and large (curve 2) grain sized electrically formed poly-Si films are presented in Fig. 1. As could be seen from the figure, with grain size increasing the peak of sensitivity is biased towards the longer waves. For the fine grain sized films the absolute sensitivity is $3.2 \mathrm{~mA} /$ $\mathrm{W}$ at the wavelength $0.5 \mu \mathrm{m}$ and its maximum rating of $10 \mathrm{~mA} / \mathrm{W}$ is observed at $\lambda=0.7 \mu \mathrm{m}$. For the large grain sized films at $\lambda=0.5 \mu \mathrm{m}$ the sensitivity is $1.2 \mathrm{~mA} / \mathrm{W}$ and its maximum rating of $11 \mathrm{~mA} / \mathrm{W}$ is reached at the wavelength $0.84 \mu \mathrm{m}$. The integrated sensitivity of poly-Si films was measured in a photo-resistive mode and for the fine grain sized films was increased from 15 up to $300 \mathrm{nA} / \mathrm{lx}$, while for the large grain sized films up to $600 \mathrm{nA} / \mathrm{lx}$.

The investigations of photocurrent properties of polySi films showed the possibility of their use both as solar cells and photoresistors rated over the wide range of spectrum. The rather high sensitivity at short waves lets their use as photodetectors in optic memory devices to read information with lasers having the wavelengths 514.5 or $632.8 \mathrm{~nm}[5]$.

\section{References}

1. M.M. Mandurah, K.C Saraswat., T.I Kamins. A model for conduction in polycrystalline silicon // IEEE Trans. Electron Devices, ED-28, p.1163-1171 (1981).

2. A.G. Abdullajev, F.D. Kasimov. Memory switching effects in the local polycrystalline silicon films // Thin Solid Films, 112, p.121-125 (1984)

3. F.D. Kasimov, Memory switching mechanism in the lightly doped polysilicon films. In: Proceedings of the 1-st All-Russian and International Conference "Actual Problems of SolidState Electronics and Microelectronics», Russia, Taganrog, p.57, 1994 (in Russian).

4. V.N. Maslov, A.J. Shick, Y.V. Shmartsev. Artificial Anomaly Photo-Voltage Structure // Fizika i Tekhn. Poluprov 14, p. 984-986 (1980) (in Russian).

5. V.A. Vul, Optical Memory Devices, Energija, Leningrad, 1979, 184 p. (in Russian). 